Supporting information for

\title{
Theoretical Study of the Methyl Transfer in Guanidinoacetate Methyltransferase
}

\author{
Polina Velichkova and Fahmi Himo* \\ Theoretical Chemistry \\ Department of Biotechnology \\ Royal Institute of Technology \\ Albanova University Center \\ SE-106 91 Stockholm \\ Sweden \\ E-mail:himo@theochem.kth.se
}

Cartesian coordinates of the reactant (Figure 2), the transition state (Figure 4), and the product (Figure 5). For the TS, the eigenvector corresponding to the imaginary frequency is also given. Atoms kept fixed in the geometry optimizations are indicated in bold face.

Total gas phase energies calculated at the B3LYP/6-311+G(2d,2p) level are also reported.

\begin{tabular}{cccc}
\multicolumn{5}{c}{ Reactant: $(\mathbf{E}=\mathbf{- 2 5 3 7 . 2 9 1 5 8 3}$ a.u. $)$} \\
C & 5.940554 & -1.374219 & -1.236124 \\
O & 6.267168 & -2.432104 & -0.333704 \\
C & $\mathbf{6 . 5 0 0 7 7 2}$ & $\mathbf{- 3 . 6 4 5 7 7 7}$ & $\mathbf{- 1 . 0 5 0 6 9 2}$ \\
C & 6.043861 & -3.403213 & -2.493948 \\
C & 6.279676 & -1.892428 & -2.643993 \\
C & 4.454600 & -1.063658 & -1.012500 \\
S & 3.972952 & 0.571836 & -1.691138 \\
C & 4.563114 & 1.628893 & -0.306456 \\
C & 4.216120 & 3.099774 & -0.465946 \\
C & $\mathbf{4 . 6 5 8 4 4 4}$ & $\mathbf{3 . 8 0 3 0 9 7}$ & $\mathbf{0 . 8 1 6 0 5 9}$ \\
C & 2.166157 & 0.527237 & -1.439475 \\
N & -0.632657 & 0.505334 & -0.411612 \\
C & -1.380271 & -0.708680 & -0.672652 \\
C & -2.333661 & -1.150461 & 0.467234 \\
O & -2.922660 & -2.257560 & 0.273765
\end{tabular}




\begin{tabular}{|c|c|c|c|}
\hline & & & \\
\hline & -0.208378 & & \\
\hline & & & \\
\hline & -2.440343 & & \\
\hline & -2.810111 & & \\
\hline & -2.125460 & & \\
\hline & & & \\
\hline & -1.03 & & \\
\hline & -3.811 & & \\
\hline & -5.09 & & \\
\hline & -6.0 & & \\
\hline & -5.7 & & \\
\hline & -6.29 & -0.5 & 577 \\
\hline & -5.71 & & \\
\hline & -5.0 & & \\
\hline & -5.8 & & \\
\hline & -7.0 & & \\
\hline & -5.5 & & -2 \\
\hline & -7.2 & & \\
\hline & 1.4 & & \\
\hline & 1.8 & & \\
\hline & 1.9 & & \\
\hline & 2.56 & & \\
\hline & 2.7 & & \\
\hline & & & \\
\hline & 4.3 & & \\
\hline & 2.8 & & \\
\hline & 3.2 & & \\
\hline & & & \\
\hline & 6.60 & & \\
\hline & 2.7 & & \\
\hline & 3.3 & & \\
\hline & $3.1 \mathrm{~S}$ & & \\
\hline & -7.0 & & \\
\hline & -4.9 & & \\
\hline & 5.9 & & \\
\hline & 4.5 & & \\
\hline & 4.02 & & \\
\hline & -1.9 & & \\
\hline & $-2.7^{\prime}$ & & \\
\hline & 1.94 & & \\
\hline & $-4.0^{\prime}$ & & \\
\hline & -4.8 & & \\
\hline & & & \\
\hline & & & \\
\hline & 1.97 & & \\
\hline & -0.66 & & \\
\hline & & & \\
\hline & & & \\
\hline & -6.154361 & -1.853287 & -2.34380 \\
\hline
\end{tabular}




$\begin{array}{rrrr}\mathrm{H} & 5.673236 & -1.428328 & -3.427131 \\ \mathrm{H} & 4.245870 & -1.036626 & 0.063697 \\ \mathrm{H} & -4.871417 & -5.305084 & -3.121310 \\ \mathrm{H} & -5.671657 & -3.893276 & -3.829547 \\ \mathrm{H} & -6.566473 & -4.947161 & -2.711072 \\ \mathrm{H} & -3.327316 & 7.004209 & -2.383624 \\ \mathrm{H} & -3.340001 & 7.039376 & -0.614569 \\ \mathrm{H} & -2.347399 & 2.998813 & -1.741243 \\ \mathrm{H} & -5.167683 & -1.508385 & 3.908016 \\ \mathrm{H} & 5.646474 & 1.475220 & -0.296502 \\ \mathrm{H} & 5.711470 & 3.604011 & 1.049996 \\ \mathrm{H} & 4.711630 & 3.519458 & -1.349875 \\ \mathrm{H} & 3.136238 & 3.226829 & -0.571041 \\ \mathrm{H} & 4.138354 & 1.202730 & 0.604678 \\ \mathrm{H} & 3.810636 & -1.798728 & -1.503236 \\ \mathrm{H} & 6.549689 & -0.504531 & -0.962264 \\ \mathrm{H} & 7.573559 & -3.884724 & -1.011968 \\ \mathrm{H} & -1.954618 & -0.622746 & -1.600756 \\ \mathrm{H} & -0.008867 & 0.448912 & 0.403574 \\ \mathrm{H} & 0.521975 & 2.624677 & 0.270474 \\ \mathrm{H} & -0.550400 & 3.769568 & -0.586261 \\ \mathrm{H} & 1.954873 & 0.232420 & -0.403379 \\ \mathrm{H} & 1.766226 & -0.178539 & -2.168615 \\ \mathrm{H} & 1.771796 & 1.526753 & -1.629313 \\ \mathrm{H} & 4.982757 & -3.647254 & -2.612773 \\ \mathrm{H} & -5.030858 & -4.280176 & -0.858063 \\ \mathrm{H} & -3.377006 & -2.838346 & -1.280139\end{array}$

Transition state: $(E=\mathbf{- 2 5 3 7 . 2 6 7 9 1 6}$ a.u. $)$

$\begin{array}{lccc}\mathrm{O} & 6.162651 & -2.686896 & -0.313668 \\ \mathbf{C} & \mathbf{6 . 2 8 2 2 1 0} & \mathbf{- 3 . 9 1 2 8 4 4} & \mathbf{- 1 . 0 4 6 5 3 8} \\ \mathrm{C} & 5.714966 & -3.622668 & -2.434790 \\ \mathrm{C} & 6.115799 & -2.154025 & -2.625200 \\ \mathrm{C} & 5.906609 & -1.591693 & -1.211430 \\ \mathrm{C} & 4.474651 & -1.111924 & -0.945604 \\ \mathrm{~S} & 4.084362 & 0.500183 & -1.730359 \\ \mathrm{C} & 4.613056 & 1.617920 & -0.366599 \\ \mathrm{C} & 4.329674 & 3.084317 & -0.672038 \\ \mathrm{C} & \mathbf{4 . 4 9 3 1 8 3} & \mathbf{3 . 8 8 9 7 2 6} & \mathbf{0 . 6 1 4 9 8 8} \\ \mathrm{C} & 1.846995 & 0.566082 & -1.256432 \\ \mathrm{~N} & -0.203160 & 0.667000 & -0.566963 \\ \mathrm{C} & -0.742960 & 1.928861 & -0.869971 \\ \mathrm{~N} & -1.715211 & 2.066957 & -1.766331 \\ \mathrm{C} & -0.926902 & -0.555255 & -0.920482 \\ \mathrm{C} & -2.165394 & -0.815627 & -0.021020 \\ \mathrm{O} & -2.406591 & 0.063264 & 0.833616 \\ \mathrm{O} & -2.776545 & -1.896898 & -0.240475 \\ \mathrm{~N} & -0.145829 & 2.978124 & -0.340130\end{array}$

Eigenvector

$\begin{array}{rrr}-0.01 & 0.02 & -0.01 \\ \mathbf{0 . 0 0} & \mathbf{0 . 0 1} & \mathbf{0 . 0 0} \\ 0.00 & 0.00 & 0.00 \\ -0.01 & 0.00 & -0.01 \\ 0.00 & 0.01 & -0.01 \\ -0.02 & -0.02 & 0.02 \\ -0.16 & 0.00 & 0.06 \\ -0.02 & 0.01 & 0.00 \\ -0.01 & 0.01 & 0.00 \\ \mathbf{0 . 0 0} & -\mathbf{0 . 0 1} & \mathbf{0 . 0 1} \\ 0.86 & -0.05 & -0.20 \\ -0.21 & 0.04 & 0.04 \\ -0.04 & -0.05 & 0.02 \\ -0.01 & 0.00 & -0.03 \\ -0.05 & 0.03 & 0.02 \\ -0.03 & -0.02 & 0.00 \\ -0.04 & -0.03 & 0.02 \\ -0.03 & -0.03 & 0.01 \\ 0.01 & -0.01 & -0.03\end{array}$




\begin{tabular}{|c|c|c|c|c|c|c|}
\hline $\mathrm{O}$ & -2.752201 & 4.460896 & -2.154190 & -0.01 & 0.03 & -0.01 \\
\hline $\mathrm{C}$ & -2.246375 & 5.428475 & -1.510925 & 0.00 & 0.01 & 0.00 \\
\hline $\mathrm{O}$ & -1.230508 & 5.390597 & -0.762536 & -0.01 & 0.02 & 0.00 \\
\hline C & -2.968503 & 6.778801 & -1.616679 & -0.01 & 0.02 & 0.00 \\
\hline $\mathrm{O}$ & 1.134521 & 0.306176 & 1.802114 & 0.05 & -0.01 & 0.01 \\
\hline $\mathrm{C}$ & 1.742064 & 1.344590 & 2.209316 & 0.02 & 0.00 & 0.00 \\
\hline $\mathrm{O}$ & 1.803826 & 2.452436 & 1.613568 & 0.03 & 0.00 & 0.00 \\
\hline $\mathrm{C}$ & 2.503945 & 1.230083 & 3.541633 & 0.00 & 0.00 & 0.02 \\
\hline $\mathrm{C}$ & 2.661746 & -0.218107 & 4.005988 & 0.01 & 0.00 & 0.00 \\
\hline $\mathrm{C}$ & 3.315581 & -1.057092 & 2.917967 & -0.01 & 0.00 & -0.01 \\
\hline $\mathrm{N}$ & 2.826763 & -2.319960 & 2.767773 & 0.00 & 0.00 & 0.00 \\
\hline C & 3.265181 & -3.175139 & 1.680389 & 0.00 & 0.00 & 0.00 \\
\hline $\mathrm{O}$ & 4.265653 & -0.633273 & 2.251263 & 0.00 & 0.00 & -0.02 \\
\hline $\mathrm{O}$ & -4.050871 & -3.143316 & -2.320924 & 0.00 & 0.00 & 0.00 \\
\hline $\mathrm{C}$ & -5.164906 & -3.709838 & -1.651072 & 0.00 & 0.00 & 0.00 \\
\hline $\mathrm{C}$ & -5.786737 & -4.728094 & -2.604893 & 0.00 & 0.00 & 0.00 \\
\hline C & -6.201338 & -2.653889 & -1.238020 & 0.00 & 0.00 & 0.00 \\
\hline $\mathrm{N}$ & -5.701535 & -1.769841 & -0.206478 & 0.00 & 0.00 & 0.00 \\
\hline $\mathrm{C}$ & -6.462294 & -0.731050 & 0.204670 & 0.00 & 0.00 & 0.00 \\
\hline $\mathrm{O}$ & -7.593656 & -0.505741 & -0.226063 & 0.00 & 0.00 & 0.00 \\
\hline C & -5.806683 & 0.214405 & 1.215115 & 0.00 & 0.00 & 0.00 \\
\hline $\mathrm{N}$ & -4.819004 & -0.364234 & 2.100343 & 0.00 & 0.00 & 0.00 \\
\hline $\mathrm{C}$ & -5.155615 & -0.976778 & 3.254225 & 0.00 & 0.00 & 0.00 \\
\hline $\mathrm{O}$ & -6.289882 & -1.122791 & 3.699140 & 0.00 & 0.00 & 0.00 \\
\hline $\mathrm{H}$ & 7.170749 & -2.082088 & -2.911147 & -0.01 & 0.01 & 0.00 \\
\hline $\mathrm{H}$ & 6.118184 & -4.285301 & -3.205795 & 0.00 & 0.01 & 0.00 \\
\hline $\mathrm{H}$ & 2.682985 & -3.007018 & 0.764543 & 0.00 & 0.00 & 0.00 \\
\hline $\mathrm{H}$ & 3.304795 & -0.265898 & 4.894937 & 0.01 & -0.01 & 0.00 \\
\hline $\mathrm{H}$ & 3.150929 & -4.224125 & 1.970530 & -0.01 & 0.00 & 0.00 \\
\hline $\mathrm{H}$ & -7.113258 & -3.149291 & -0.877475 & 0.00 & 0.00 & 0.00 \\
\hline $\mathrm{H}$ & -5.325183 & 1.022638 & 0.653680 & 0.00 & 0.00 & 0.00 \\
\hline $\mathrm{H}$ & 5.743597 & -4.697231 & -0.505098 & -0.01 & 0.02 & 0.01 \\
\hline $\mathrm{H}$ & 4.386980 & 4.961478 & 0.415951 & 0.00 & -0.01 & 0.00 \\
\hline $\mathrm{H}$ & 3.723111 & 3.597000 & 1.333060 & -0.01 & -0.01 & -0.01 \\
\hline $\mathrm{H}$ & -2.247957 & 7.600676 & -1.596907 & -0.01 & 0.02 & 0.00 \\
\hline $\mathrm{H}$ & -2.308283 & 1.270964 & -1.941631 & -0.10 & 0.04 & 0.08 \\
\hline $\mathrm{H}$ & 1.905483 & -2.487654 & 3.145015 & 0.00 & 0.01 & -0.01 \\
\hline $\mathrm{H}$ & -3.834638 & -0.283464 & 1.808572 & 0.00 & -0.01 & -0.02 \\
\hline $\mathrm{H}$ & -4.720441 & -1.831102 & 0.069614 & 0.01 & 0.01 & -0.02 \\
\hline $\mathrm{H}$ & 1.689042 & -0.638036 & 4.276256 & 0.01 & 0.00 & 0.00 \\
\hline $\mathrm{H}$ & 3.483305 & 1.699609 & 3.415877 & 0.00 & 0.01 & 0.03 \\
\hline $\mathrm{H}$ & 1.965687 & 1.816802 & 4.295016 & 0.00 & -0.01 & 0.02 \\
\hline $\mathrm{H}$ & -0.238700 & -1.396289 & -0.791110 & -0.02 & 0.05 & 0.00 \\
\hline $\mathrm{H}$ & 4.315119 & -2.971631 & 1.468724 & 0.00 & -0.01 & 0.01 \\
\hline $\mathrm{H}$ & -6.623636 & 0.644484 & 1.799153 & 0.00 & 0.00 & 0.00 \\
\hline $\mathrm{H}$ & -6.490782 & -2.076613 & -2.126997 & 0.00 & 0.00 & 0.00 \\
\hline $\mathrm{H}$ & 5.527090 & -1.627065 & -3.380788 & 0.00 & -0.01 & -0.02 \\
\hline $\mathrm{H}$ & 4.323085 & -0.998634 & 0.132192 & -0.02 & -0.01 & 0.03 \\
\hline $\mathrm{H}$ & -5.034706 & -5.464727 & -2.901886 & 0.00 & 0.00 & 0.00 \\
\hline $\mathrm{H}$ & -6.142964 & -4.225571 & -3.510916 & 0.00 & 0.00 & 0.00 \\
\hline
\end{tabular}




$\begin{array}{rrrrrrrr}\mathrm{H} & -6.630235 & -5.250460 & -2.141744 & 0.00 & 0.00 & 0.00 \\ \mathrm{H} & -3.582135 & 6.825682 & -2.519160 & -0.01 & 0.02 & 0.00 \\ \mathrm{H} & -3.626988 & 6.894895 & -0.748005 & -0.01 & 0.02 & 0.00 \\ \mathrm{H} & -2.129623 & 3.053555 & -1.930486 & 0.03 & -0.03 & -0.02 \\ \mathrm{H} & -4.258325 & -1.347171 & 3.793533 & 0.00 & 0.00 & -0.01 \\ \mathrm{H} & 5.682174 & 1.446472 & -0.209614 & -0.01 & -0.06 & -0.12 \\ \mathrm{H} & 5.484104 & 3.733838 & 1.059447 & 0.00 & -0.01 & 0.01 \\ \mathrm{H} & 4.999323 & 3.443131 & -1.463283 & 0.00 & 0.00 & 0.00 \\ \mathrm{H} & 3.304405 & 3.213893 & -1.033926 & & -0.01 & 0.02 & 0.02 \\ \mathrm{H} & 4.081755 & 1.293974 & 0.531062 & & 0.03 & 0.00 & 0.03 \\ \mathrm{H} & 3.748442 & -1.831806 & -1.334828 & -0.02 & -0.01 & 0.01 \\ \mathrm{H} & 6.614840 & -0.789913 & -0.972450 & & 0.00 & 0.01 & -0.01 \\ \mathrm{H} & 7.341939 & -4.203955 & -1.106105 & & 0.00 & 0.02 & 0.01 \\ \mathrm{H} & -1.213196 & -0.545870 & -1.977261 & -0.07 & 0.01 & 0.02 \\ \mathrm{H} & 0.126756 & 0.617923 & 0.428774 & 0.00 & 0.03 & -0.09 \\ \mathrm{H} & 0.571781 & 2.825749 & 0.389386 & -0.06 & 0.00 & 0.00 \\ \mathrm{H} & -0.564579 & 3.944143 & -0.501212 & 0.00 & -0.04 & -0.01 \\ \mathrm{H} & 2.000413 & 0.158339 & -0.265422 & 0.00 & 0.07 & 0.00 \\ \mathrm{H} & 1.553610 & -0.070464 & -2.078553 & 0.19 & -0.03 & 0.02 \\ \mathrm{H} & 1.792897 & 1.636229 & -1.382458 & 0.05 & -0.06 & -0.05 \\ \mathrm{H} & 4.625428 & -3.734702 & -2.438197 & 0.00 & 0.00 & -0.01 \\ \mathrm{H} & -4.834967 & -4.237866 & -0.738766 & 0.00 & 0.00 & 0.00 \\ \mathrm{H} & -3.500251 & -2.677834 & -1.656214 & 0.00 & 0.01 & 0.01\end{array}$

Product: ( $E=\mathbf{- 2 5 3 7 . 3 4 9 2 5 7}$ a.u. $)$

$\begin{array}{lccc}\mathrm{O} & 6.133633 & -3.232436 & -0.338838 \\ \mathrm{C} & \mathbf{5 . 7 3 0 2 3 5} & \mathbf{- 4 . 0 9 9 8 0 5} & \mathbf{- 1 . 4 0 4 2 8 7} \\ \mathrm{C} & 5.605220 & -3.221643 & -2.652825 \\ \mathrm{C} & 6.672232 & -2.151330 & -2.384038 \\ \mathrm{C} & 6.565616 & -1.955738 & -0.861085 \\ \mathrm{C} & 5.552494 & -0.896581 & -0.427650 \\ \mathrm{~S} & 6.074023 & 0.793475 & -0.931088 \\ \mathrm{C} & 5.055116 & 1.764474 & 0.258020 \\ \mathrm{C} & 4.922744 & 3.218573 & -0.189947 \\ \mathrm{C} & \mathbf{4 . 1 6 6 4 1 7} & \mathbf{4 . 0 5 1 6 3 5} & \mathbf{0 . 8 4 8 4 9 5} \\ \mathrm{C} & 1.174045 & 0.645025 & -1.569262 \\ \mathrm{~N} & -0.259079 & 0.835507 & -1.753395 \\ \mathrm{C} & -0.832117 & 2.077986 & -1.719413 \\ \mathrm{~N} & -2.033477 & 2.280141 & -2.245693 \\ \mathrm{C} & -1.098326 & -0.349546 & -1.833171 \\ \mathrm{C} & -2.121148 & -0.519418 & -0.678048 \\ \mathrm{O} & -1.969064 & 0.148960 & 0.384018 \\ \mathrm{O} & -3.038202 & -1.341300 & -0.904818 \\ \mathrm{~N} & -0.154364 & 3.109572 & -1.200311 \\ \mathrm{O} & -3.358444 & 4.471092 & -1.768555 \\ \mathrm{C} & -2.693087 & 5.448700 & -1.306368 \\ \mathrm{O} & -1.453513 & 5.482105 & -1.084398 \\ \mathrm{C} & \mathbf{- 3 . 5 0 0 1 9 7} & \mathbf{6 . 6 9 9 7 9 1} & \mathbf{- 0 . 9 4 4 3 0 8} \\ \mathrm{O} & -0.032193 & 0.269094 & 2.057417\end{array}$




\begin{tabular}{|c|c|c|c|}
\hline $\mathrm{C}$ & 0.906036 & 1.198822 & 2.137691 \\
\hline $\mathrm{O}$ & 1.103433 & 2.079716 & 1.310529 \\
\hline $\mathrm{C}$ & 1.741023 & 1.088894 & 3.405833 \\
\hline $\mathrm{C}$ & 2.009729 & -0.346865 & 3.875542 \\
\hline $\mathrm{C}$ & 2.958730 & -1.077900 & 2.927317 \\
\hline $\mathrm{N}$ & 2.533526 & -2.297235 & 2.496714 \\
\hline C & 3.301629 & -3.110033 & 1.568245 \\
\hline $\mathrm{O}$ & 4.045520 & -0.594914 & 2.609929 \\
\hline $\mathrm{O}$ & -4.481099 & -2.925586 & -2.632181 \\
\hline $\mathrm{C}$ & -5.201488 & -3.698285 & -1.684458 \\
\hline $\mathrm{C}$ & -5.826213 & -4.863229 & -2.449434 \\
\hline C & -6.286316 & -2.881822 & -0.966122 \\
\hline $\mathrm{N}$ & -5.737391 & -1.920661 & -0.033690 \\
\hline $\mathrm{C}$ & -6.574281 & -1.194131 & 0.736523 \\
\hline $\mathrm{O}$ & -7.799415 & -1.318796 & 0.723753 \\
\hline $\mathrm{C}$ & -5.925335 & -0.132763 & 1.624843 \\
\hline $\mathrm{N}$ & -4.514692 & -0.293913 & 1.934297 \\
\hline $\mathrm{C}$ & -4.093686 & -0.861580 & 3.091442 \\
\hline $\mathrm{O}$ & -4.805904 & -1.272834 & 3.999755 \\
\hline $\mathrm{H}$ & 7.665361 & -2.533535 & -2.645336 \\
\hline $\mathrm{H}$ & 5.773339 & -3.778842 & -3.578986 \\
\hline $\mathrm{H}$ & 3.042441 & -2.900960 & 0.522964 \\
\hline $\mathrm{H}$ & 2.509226 & -0.312140 & 4.850689 \\
\hline $\mathrm{H}$ & 3.114701 & -4.168668 & 1.769175 \\
\hline $\mathrm{H}$ & -6.958844 & -3.552664 & -0.417231 \\
\hline $\mathrm{H}$ & -6.083684 & 0.839625 & 1.143817 \\
\hline $\mathrm{H}$ & 4.790701 & -4.589144 & -1.123285 \\
\hline $\mathrm{H}$ & 4.031774 & 5.082385 & 0.504351 \\
\hline $\mathrm{H}$ & 3.175950 & 3.630359 & 1.047256 \\
\hline $\mathrm{H}$ & -2.874037 & 7.593520 & -0.999630 \\
\hline $\mathrm{H}$ & -2.473115 & 1.542663 & -2.771870 \\
\hline $\mathrm{H}$ & 1.571589 & -2.546445 & 2.672371 \\
\hline $\mathrm{H}$ & -3.779686 & -0.017409 & 1.282093 \\
\hline $\mathrm{H}$ & -4.745477 & -1.689947 & -0.084463 \\
\hline $\mathrm{H}$ & 1.066544 & -0.883964 & 4.002696 \\
\hline $\mathrm{H}$ & 2.682928 & 1.610990 & 3.231429 \\
\hline $\mathrm{H}$ & 1.197653 & 1.632011 & 4.189135 \\
\hline $\mathrm{H}$ & -0.442023 & -1.224442 & -1.818337 \\
\hline $\mathrm{H}$ & 4.362618 & -2.902089 & 1.695907 \\
\hline $\mathrm{H}$ & -6.479204 & -0.133617 & 2.565338 \\
\hline $\mathrm{H}$ & -6.899900 & -2.378304 & -1.726876 \\
\hline $\mathrm{H}$ & 6.509515 & -1.223842 & -2.938612 \\
\hline $\mathrm{H}$ & 5.441190 & -0.914048 & 0.658957 \\
\hline $\mathrm{H}$ & -5.046876 & -5.418128 & -2.979245 \\
\hline $\mathrm{H}$ & -6.537109 & -4.487342 & -3.193465 \\
\hline $\mathrm{H}$ & -6.354069 & -5.547689 & -1.777508 \\
\hline $\mathrm{H}$ & -4.372768 & 6.800793 & -1.594574 \\
\hline $\mathrm{H}$ & -3.861245 & 6.602061 & 0.086390 \\
\hline $\mathrm{H}$ & -2.578325 & 3.203087 & -2.046374 \\
\hline $\mathrm{H}$ & -2.987078 & -0.901068 & 3.135793 \\
\hline
\end{tabular}




$\begin{array}{crrc}\mathrm{H} & 5.515369 & 1.695969 & 1.248390 \\ \mathrm{H} & 4.713502 & 4.087171 & 1.798057 \\ \mathrm{H} & 5.918435 & 3.647901 & -0.363388 \\ \mathrm{H} & 4.396843 & 3.252070 & -1.151685 \\ \mathrm{H} & 4.067956 & 1.298131 & 0.329438 \\ \mathrm{H} & 4.571109 & -1.115690 & -0.863489 \\ \mathrm{H} & 7.539244 & -1.723124 & -0.413769 \\ \mathrm{H} & 6.490327 & -4.883749 & -1.548160 \\ \mathrm{H} & -1.647317 & -0.411498 & -2.780834 \\ \mathrm{H} & -0.679849 & 0.370691 & 1.277509 \\ \mathrm{H} & 0.408112 & 2.891175 & -0.382395 \\ \mathrm{H} & -0.645848 & 4.045176 & -1.199247 \\ \mathrm{H} & 1.415910 & 0.249297 & -0.577498 \\ \mathrm{H} & 1.545661 & -0.052224 & -2.329294 \\ \mathrm{H} & 1.689960 & 1.596347 & -1.694785 \\ \mathrm{H} & 4.609480 & -2.770011 & -2.710804 \\ \mathrm{H} & -4.516871 & -4.103758 & -0.918729 \\ \mathrm{H} & -3.892576 & -2.316739 & -2.144038\end{array}$

